\title{
Domain Bubbles of Extra Dimensions
}

\author{
J.R. Morrist \\ Physics Dept., Indiana University Northwest, \\ 3400 Broadway, Gary, Indiana 46408
}

\begin{abstract}
"Dimension bubbles" of the type previously studied by Blau and Guendelman [S.K. Blau and E.I. Guendelman, Phys. Rev. D40, 1909 (1989)], which effectively enclose a region of $5 \mathrm{~d}$ spacetime and are surrounded by a region of $4 \mathrm{~d}$ spacetime, can arise in a $5 \mathrm{~d}$ theory with a compact extra dimension that is dimensionally reduced to give an effective $4 \mathrm{~d}$ theory. These bubbles with thin domain walls can be stabilized against total collapse in a rather natural way by a scalar field which, as in the case with "ordinary" nontopological solitons, traps light scalar particles inside the bubble.
\end{abstract}

PACS: 11.27.+d, 04.50.+h, 98.80.Cq

*E-mail: jmorris@iun.edu 


\section{INTRODUCTION}

Blau and Guendelmann [1] have investigated the interesting case of an inhomogeneous spacetime formed by sewing together regions having different numbers of macroscopic spatial dimensions. From a four dimensional (4d) point of view the scale factor of a fifth (compact) dimension can be treated as a scalar field which is allowed to vary rapidly in a localized region of the $4 \mathrm{~d}$ spacetime, forming a domain wall. The domain wall can thereby interpolate between two regions differing in the number of macroscopic space dimensions. Closed domain walls can form "dimension bubbles" with different numbers of macroscopic space dimensions on the interior and exterior of the bubble wall. As examples, Blau and Guendelmann [1] have used the low temperature Rubin-Roth $4 \mathrm{~d}$ effective potential, generated by including oneloop corrections due to quantum fluctuations from fermions and bosons [2]. Fermions, which can have Casimir energies of opposite sign from bosonic ones, can act toward stabilizing a compact extra dimension [2] from collapsing due to the gravitational Casimir effect [3]. From a $4 \mathrm{~d}$ point of view, the domain wall arises from the scalar dilaton field $\varphi$ associated with the scale factor of the extra dimension, and the Rubin-Roth potential becomes a function of this field.

There is a range of parameters for which the $4 \mathrm{~d}$ effective potential $U(\varphi)$ exhibits one local minimum, at say, $\varphi=\varphi_{\text {min }}$, separated by a potential barrier from another minimum approached asymptotically as $\varphi \rightarrow \infty$. This type of potential gives rise to what will be referred to here as a "semi-vacuumless" domain wall, since it seems to be a hybrid between an ordinary domain wall and a "vacuumless" domain wall of the type originally described by Vilenkin and Cho [4] and further studied by Bazeia [5]. The scalar field $\varphi$ interpolates between the vacuum domain $\left(\varphi=\varphi_{\text {min }}\right)$ and the "vacuumless" domain $(\varphi \rightarrow \infty)$. If the values of the effective potential $U(\varphi)$ differ at all on the two different sides of the wall, the wall will generally be unstable against bending. As a result, a network of closed domain bubbles can form. Such a bubble, however, will be unstable against collapse without a mechanism to balance the inward pressure due to the tension in the bubble wall.

A simple mechanism proposed here to stabilize one of these "semi-vacuumless" bubbles consists of the inclusion of a fundamental scalar field $\chi$ in the original $5 \mathrm{~d}$ action. Upon dimensional reduction to the effective $4 \mathrm{~d}$ (Einstein frame) theory, this field $\chi$ is seen to have a mass which depends upon $\varphi$, so that lower mass $\chi$ bosons in the interior of the bubble can be effectively trapped, as is the case with an "ordinary" nontopological soliton (NTS), as

previously studied by Frieman, Gleiser, Gelmini and Kolb [6]. The trapped $\chi$ particles give rise to an outward pressure that can stabilize the bubble from total collapse. We specifically consider bubbles, in the thin wall approximation, with $\varphi=\varphi_{\min }$ outside the bubble and 
$\varphi=\varphi_{1} \gg \varphi_{\min }$ inside the bubble. The scale factor $B$ of the extra dimension is related to the scalar $\varphi$ by $B=e^{\sqrt{\frac{2}{3}} \kappa \varphi}$, with $\kappa=\sqrt{8 \pi G}$, so that for $\varphi_{1} \rightarrow \infty$ the extra dimension becomes macroscopic in the bubble's interior, even if the bubble has a small size in the surrounding $3 \mathrm{~d}$ space. This object is then a "dimension bubble" embedded in an effective $4 \mathrm{~d}$ spacetime with an effective 5d interior (for a macroscopic bubble). (A microscopic bubble would have only one macroscopically large space dimension inside.) As with ordinary NTSs, these bubbles, if physically realized, could contribute to the dark matter content of our universe.

The rest of the paper is organized in the following way: The dimensional reduction of the $5 \mathrm{~d}$ action is presented in sec. 2, allowing an extraction of the basic form of the $4 \mathrm{~d}$ effective potential. The $4 \mathrm{~d}$ effective potential is written in terms of the Rubin-Roth potential and a 5d cosmological constant. The contributions to the low temperature Rubin-Roth potential are listed, which describe contributions from quantum fluctuations for bosons and fermions. Semi-vacuumless domain walls arising from the $4 \mathrm{~d}$ effective potential along with the instability of dimension bubbles are considered in sec. 3. The stabilization mechanism is presented in sec. 4, describing $\chi$-boson stabilized NTS bags. Some properties of these NTS bags, such as the bag mass and limits on the bag charge and radius, are investigated. A brief summary forms sec. 5 .

\section{DIMENSIONAL REDUCTION OF THE FIVE-DIMENSIONAL ACTION}

\section{A. Metric Ansatz}

We begin by considering a five-dimensional spacetime described by a metric $\tilde{g}_{M N}$ :

$$
d s^{2}=\tilde{g}_{M N} d x^{M} d x^{N}=\tilde{g}_{\mu \nu} d x^{\mu} d x^{\nu}+\tilde{g}_{55} d y^{2}
$$

where $x^{M}=\left(x^{\mu}, y\right)$, with $M, N=0, \cdots 3,5$ and $\mu, \nu=0, \cdots 3$. We denote $\tilde{g}=\operatorname{det} \tilde{g}_{\mu \nu}$, and $\tilde{g}_{5}=\operatorname{det} \tilde{g}_{M N}$, so that $\sqrt{\left|\tilde{g}_{5}\right|}=\sqrt{-\tilde{g}} \sqrt{\left|\tilde{g}_{55}\right|}$. The extra dimension will be assumed to be toroidally compact, so that the 5 d spacetime has topology of $M_{4} \times S^{1}$.

We assume an ansatz where the metric $\tilde{g}_{M N}$ is independent of the extra dimension $y$, i.e., $g_{M N}=g_{M N}\left(x^{\mu}\right), \partial_{5} g_{M N}=0$, and the metric factorizes with $g_{\mu n}=0$. The extra dimension is described by a linear coordinate $y$ lying in the range $0 \leq y \leq 2 \pi R$ and can be assigned a (dimensionless) scale factor $B\left(x^{\mu}\right)$ with $\tilde{g}_{55}=-B^{2}$. A scalar field $\varphi$ can be defined by

$$
\varphi=\frac{1}{\kappa} \sqrt{\frac{3}{2}} \ln B,
$$

where $\kappa$ is related to the 4 d Planck mass $M_{P}$ by $\kappa=\sqrt{8 \pi G}=\sqrt{8 \pi} M_{P}^{-1}$, so that the scale factor can be written as $B=e^{\sqrt{\frac{2}{3}} \kappa \varphi}$. 


\section{B. The 5d Action and Dimensional Reduction}

We take the 5-dimensional action to include the $5 \mathrm{~d}$ Einstein action, cosmological constant $\Lambda$, and a source Lagrangian $\mathcal{L}_{5}$ :

$$
\begin{aligned}
S_{5} & =\frac{1}{2 \kappa_{5}^{2}} \int d^{5} x \sqrt{\tilde{g}_{5}}\left\{\tilde{R}_{5}-2 \Lambda+2 \kappa_{5}^{2} \mathcal{L}_{5}\right\} \\
& =\frac{V_{y}}{2 \kappa_{5}^{2}} \int d^{4} x \sqrt{-\tilde{g}}(B)\left\{\tilde{R}_{5}-2 \Lambda+2 \kappa_{5}^{2} \mathcal{L}_{5}\right\} \\
& =\frac{1}{2 \kappa^{2}} \int d^{4} x \sqrt{-\tilde{g}}(B)\left\{\tilde{R}_{5}-2 \Lambda+2 \kappa^{2} \mathcal{L}\right\}
\end{aligned}
$$

where we have used the definitions $V_{y}=\int d y=(2 \pi R), \kappa_{5}^{2}=8 \pi G_{5}=V_{y} \kappa^{2}$, and $\mathcal{L}=V_{y} \mathcal{L}_{5}=$ $(2 \pi R) \mathcal{L}_{5}$. In addition, $\tilde{R}_{5}=\tilde{g}^{M N} \tilde{R}_{M N}$ denotes the 5 -dimensional Ricci scalar built from $\tilde{g}_{M N}$. Note that the $4 \mathrm{~d}$ Jordan Frame metric is $\tilde{g}_{\mu \nu}$, the $\mu \nu$ part of $\tilde{g}_{M N}$. A $4 \mathrm{~d}$ Einstein Frame metric $g_{\mu \nu}$ can be defined by $g_{\mu \nu}=B \tilde{g}_{\mu \nu}=e^{\sqrt{\frac{2}{3}} \kappa \varphi} \tilde{g}_{\mu \nu}$, in which case the line element in (1) takes the Kaluza-Klein form

$$
\begin{aligned}
d s^{2} & =B^{-1} g_{\mu \nu} d x^{\mu} d x^{\nu}-B^{2} d y^{2} \\
& =e^{-\sqrt{\frac{2}{3}} \kappa \varphi} g_{\mu \nu} d x^{\mu} d x^{\nu}-e^{2 \sqrt{\frac{2}{3}} \kappa \varphi} d y^{2}
\end{aligned}
$$

Using (3) and (4), the $5 \mathrm{~d}$ action is dimensionally reduced to the effective $4 \mathrm{~d}$ Einstein Frame action

$$
S=\int d^{4} x \sqrt{-g}\left\{\frac{1}{2 \kappa^{2}} R+\frac{1}{2}(\nabla \varphi)^{2}+e^{-\sqrt{\frac{2}{3}} \kappa \varphi}\left[\mathcal{L}-\frac{1}{\kappa^{2}} \Lambda\right]\right\}
$$

where $R=g^{\mu \nu} R_{\mu \nu}$ is the $4 \mathrm{~d}$ Ricci scalar built from the $4 \mathrm{~d}$ Einstein Frame metric $g_{\mu \nu}$ and $g=\operatorname{det} g_{\mu \nu}$. [We use a $4 \mathrm{~d}$ metric with signature $(+,-,-,-)$.]

\section{The Form of the 4d Effective Potential}

In order to investigate the effects of Casimir-like one loop quantum corrections on the size of the extra dimension, we follow Blau and Guendelman [1] and use the low temperature limit of the finite-temperature Rubin-Roth potential $V_{R R}$ for bosons and fermions [2]. From this potential, along with a cosmological constant term, a $4 \mathrm{~d}$ effective potential $U$ can be constructed. The part of the original action containing the potential terms, from (3), is

$$
S_{5, \mathrm{pot}}=-\int d^{5} x \sqrt{\tilde{g}_{5}}\left\{V_{5}+\frac{\Lambda}{\kappa_{5}^{2}}\right\}=-\int d^{4} x \sqrt{-\tilde{g}_{4}} \int d y\left|\tilde{g}_{55}\right|^{1 / 2}\left\{V_{5}+\frac{\Lambda}{\kappa_{5}^{2}}\right\}
$$

where $V_{5}$ is the potential appearing in $\mathcal{L}_{5}=\mathcal{L} /(2 \pi R)$. Using (5), we identify the $4 \mathrm{~d}$ Einstein Frame effective potential $U$ as 


$$
U=\frac{1}{B}\left[V+\frac{\Lambda}{\kappa^{2}}\right]=e^{-\sqrt{\frac{2}{3}} \kappa \varphi}\left[V+\frac{\Lambda}{\kappa^{2}}\right]
$$

where $V=V_{5}(2 \pi R)$ is the potential term appearing in $\mathcal{L}$. Identifying the Rubin-Roth potential through the relation

$$
V_{R R} \propto \int d y\left|\tilde{g}_{55}\right|^{1 / 2} V_{5}=B V
$$

so that $V \propto \frac{V_{R R}}{B}$, allows the 4 d effective potential to be written in terms of $V_{R R}$ as 1

$$
U \propto \frac{1}{B}\left[\frac{V_{R R}}{B}+\frac{\Lambda}{\kappa^{2}}\right]=e^{-\sqrt{\frac{2}{3}} \kappa \varphi}\left[e^{-\sqrt{\frac{2}{3}} \kappa \varphi} V_{R R}+\frac{\Lambda}{\kappa^{2}}\right]
$$

\section{Low Temperature Rubin-Roth Potential (per degree of freedom)}

Following the example of Blau and Guendelman, we consider a potential with an interesting structure generated by Casimir contributions from the graviton and additional fermionic matter fields [四]. We are interested in the limit of negligible temperature, i.e., the high $\beta=1 / T$ limit. The asymptotic behaviors of bosonic and fermionic contributions to the Rubin-Roth potential (per degree of freedom) are listed in Table 1 of ref. [2]. The results for massless bosons and massive fermions are listed here for convenience. The potential terms can be written in terms of the circumference of the extra dimension, $L_{5}=(2 \pi R) B$.

Massless Bosons For massless bosons, we have (for all $L_{5}$ ),

$$
V_{R R}^{(b)} \sim-\frac{3 \zeta(5)}{4 \pi^{2}} \frac{\beta}{L_{5}^{4}}
$$

where the scale factor $B$ is related to $L_{5}$ by

$$
\left(\frac{L_{5}}{2 \pi R}\right)=\left|\tilde{g}_{55}\right|^{1 / 2}=B=e^{\sqrt{\frac{2}{3}} \kappa \varphi}
$$

Fermions $(M \geq 0) \quad$ We can consider the low temperature (high $\beta$ ) limit with $L_{5} \ll \beta$ and $\beta \gg 1 / M$ (i.e., $T \ll M$, for $M>0)$ and the two cases of (1)small $L_{5}\left(L_{5} \ll 1 / M\right)$ and (2)large $L_{5}\left(L_{5} \gg 1 / M\right)$ :

\footnotetext{
${ }^{1}$ The Rubin-Roth potential $V_{R R}$ is calculated in the Jordan Frame with a Minkowski background in ref. [2]. A factor of $B^{-2}=\exp \left(-2 \sqrt{\frac{2}{3}} \kappa \varphi\right)$ then accompanies $V_{R R}$ in the expression for the Einstein Frame effective potential $U$. See ref. [1] for a basic description of the effective potential and its dependence upon parameters.
} 


$$
V_{R R}^{(f)} \sim\left\{\begin{array}{ll}
\frac{3 \zeta(5)}{4 \pi^{2}} \frac{\beta}{L_{5}^{4}}, & L_{5} \ll \frac{1}{M}, M \geq 0 \\
\frac{M^{2}}{4 \pi^{2}} \frac{\beta}{L_{5}^{2}} e^{-M L_{5}}, & L_{5} \gg \frac{1}{M}, M>0
\end{array}\right\}
$$

Assume now that there are $N_{b}$ massless bosonic degrees of freedom with $N_{b} \geq 5$, (5 graviton degrees of freedom) since, at least the graviton, contributes and $N_{f}$ fermionic degrees of freedom, with $N_{f}>N_{b}$ (as in ref. [1]). We then write the net Rubin-Roth potential as

$$
V_{R R}=N_{b} V_{R R}^{(b)}+N_{f} V_{R R}^{(f)}
$$

so that, with the help of (10) and (12), we have

$$
V_{R R} \sim\left\{\begin{array}{ll}
\left(N_{f}-N_{b}\right) \frac{3 \zeta(5)}{4 \pi^{2}} \frac{\beta}{L_{5}^{4}}, & L_{5} \ll \frac{1}{M} \\
-N_{b} \frac{3 \zeta(5)}{4 \pi^{2}} \frac{\beta}{L_{5}^{4}}+N_{f} \frac{M^{2}}{4 \pi^{2}} \frac{\beta}{L_{5}^{2}} e^{-M L_{5}}, & L_{5} \gg \frac{1}{M}
\end{array}\right\}
$$

Note: For fermions with different masses, we can make the replacement

$$
N_{f} \frac{M^{2}}{4 \pi^{2}} \frac{\beta}{L_{5}^{2}} e^{-M L_{5}} \rightarrow \sum_{i}\left\{N_{f}^{(i)} \frac{M_{i}^{2}}{4 \pi^{2}} \frac{\beta}{L_{5}^{2}} e^{-M_{i} L_{5}}\right\}
$$

with the index $i$ running over the different fermionic species.

\section{E. The Four Dimensional Effective Potential}

Using (9) and (11) the 4d Einstein Frame effective potential $U$ can be written in terms of the circumference of the extra dimension, $L_{5}$, as

$$
U=c_{1}^{\prime} \frac{V_{R R}}{L_{5}^{2}}+\frac{c_{2}}{L_{5}} \frac{\Lambda}{\kappa^{2}}
$$

where $c_{1}^{\prime}$ and $c_{2}$ are positive constants. Using the asymptotic forms of (14) the effective potential takes the form

$$
U \sim\left\{\begin{array}{ll}
c_{1}\left(N_{f}-N_{b}\right) \frac{3 \zeta(5)}{4 \pi^{2}} \frac{\beta}{L_{5}^{6}}+c_{2} \frac{\Lambda}{\kappa^{2} L_{5}}, & L_{5} \ll \frac{1}{M} \\
-c_{1} N_{b} \frac{3 \zeta(5)}{4 \pi^{2}} \frac{\beta}{L_{5}^{6}}+c_{1} N_{f} \frac{M^{2}}{4 \pi^{2}} \frac{\beta}{L_{5}^{4}} e^{-M L_{5}}+c_{2} \frac{\Lambda}{\kappa^{2} L_{5}}, & L_{5} \gg \frac{1}{M}
\end{array}\right\}
$$

Again, for the case of nondegenerate fermions, one can make the replacement given in (15).

Equivalently, by (11), the effective potential can be written in terms of the scalar field $\varphi$, 


$$
U \sim\left\{\begin{array}{lc}
C_{1}\left(N_{f}-N_{b}\right) \frac{3 \zeta(5)}{4 \pi^{2}} \beta e^{-6 \sqrt{\frac{2}{3}} \kappa \varphi}+C_{2} \frac{\Lambda}{\kappa^{2}} e^{-\sqrt{\frac{2}{3}} \kappa \varphi}, & e^{L_{5} \ll \frac{1}{M}, \text { or }} \\
-C_{1} N_{b} \frac{3 \zeta(5)}{4 \pi^{2}} \beta e^{-6 \sqrt{\frac{2}{3}} \kappa \varphi} & \\
+\tilde{C}_{1} N_{f} \frac{M^{2}}{4 \pi^{2}} \beta e^{\left[-M(2 \pi R) \exp \left(\sqrt{\frac{2}{3}} \kappa \varphi\right)\right]}+C_{2} \frac{\Lambda}{\kappa^{2}} e^{-\sqrt{\frac{2}{3}} \kappa \varphi}, & e^{\sqrt{\frac{2}{3}} \kappa \varphi} \gg \frac{1}{M}, \text { or } \\
& \frac{L_{5} \pi R}{2 \pi R}
\end{array}\right\}
$$

with the replacement (15) for nondegenerate fermions

$$
N_{f} M^{2} e^{-M(2 \pi R) \exp \left(\sqrt{\frac{2}{3}} \kappa \varphi\right)} \rightarrow \sum_{i}\left\{N_{f}^{(i)} M_{i}^{2} e^{-M_{i}(2 \pi R) \exp \left(\sqrt{\frac{2}{3}} \kappa \varphi\right)}\right\}
$$

\section{III. "SEMI-VACUUMLESS" DOMAIN WALLS AND BUBBLES}

\section{A. The Assumed Form of the Effective Potential $U$}

In what follows we assume that the potential parameters allow the low temperature 4d effective potential to take a form (see [1]) such that $U$ has a local minimum at some finite value $L_{5, \min }$ (corresponding to $\varphi=\varphi_{\min }$ ), a local maximum at some finite $L_{5, \max }>L_{5, \min }$ (corresponding to $\varphi=\varphi_{\max }>\varphi_{\min }$ ), and asymptotically, $U \rightarrow 0$ as $L_{5} \rightarrow \infty(\varphi \rightarrow \infty)$. Therefore, there is a vacuum state at $\varphi=\varphi_{\min }$ where $U=U\left(\varphi_{\min }\right)$ and another low energy state as $\varphi \rightarrow \infty$, where $U \rightarrow 0$. These two low energy states are separated by a potential barrier at $\varphi=\varphi_{\max }$ where $U=U\left(\varphi_{\max }\right)$. We can think of this type of system as being "semi-vacuumless", since it is a hybrid of a "vacuumless" system (see, e.g., [4.5) and a system with an ordinary vacuum state.

\section{B. The "Semi-vacuumless" Domain Wall}

The potential described above allows the formation of a domain wall that interpolates between the vacuum domain $\left(\varphi=\varphi_{\text {min }}\right)$ and the "vacuumless" domain $(\varphi \rightarrow \infty)$. We will refer to this type of domain wall as a "semi-vacuumless" domain wall. This type of domain wall differs from the ordinary $\varphi$ "kink" type [and from a symmetric "vacuumless" type (see, e.g., [四, [0] ) in that there is no discrete symmetry associated with the potential, i.e., the potential and the domain wall are asymmetric. Furthermore, assuming that in the "vacuumless" domain the scalar field assumes a finite value $\varphi_{1}$ with $U\left(\varphi_{1}\right)>0$, it is most likely that the two low energy states are nondegenerate, with $U\left(\varphi_{\min }\right) \neq U\left(\varphi_{1}\right)$.

Let us consider a static, planar domain wall lying in the $y-z$ plane described by $\varphi=$ $\varphi(x)$. The $4 \mathrm{~d}$ Einstein Frame effective Lagrangian for $\varphi$ is $\mathcal{L}_{\varphi}=\frac{1}{2}(\partial \varphi)^{2}-U(\varphi)$ and the 
associated energy-momentum tensor is $T_{\mu \nu}=\partial_{\mu} \varphi \partial_{\nu} \varphi-\eta_{\mu \nu}\left[\frac{1}{2}(\partial \varphi)^{2}-U\right]$. This gives energy density and stress components $T_{00}=\frac{1}{2} \varphi^{\prime 2}+U, T_{11}=\frac{1}{2} \varphi^{\prime 2}-U$, and $T_{22}=T_{33}=-T_{00}=$ $-\left(\frac{1}{2} \varphi^{\prime 2}+U\right)$, where $\varphi^{\prime}=\partial \varphi / \partial x$. In a flat Minkowski background the equation of motion gives $\frac{1}{2} \varphi^{\prime 2}=U+K$, where $K$ is a constant of integration. The energy density and stress components of the domain wall configuration can then be written as

$$
T_{11}=K, \quad T_{22}=T_{33}=-T_{00}=-(2 U+K)
$$

\section{Unstable Domain Bubbles of Extra Dimensions}

With the assumption that the domain wall connects two different spatial regions where $\varphi \approx \varphi_{\min }$ on one side of the wall and $\varphi \approx \varphi_{1}$ on the other side, with $U\left(\varphi_{\min }\right) \neq U\left(\varphi_{1}\right)$, (20) shows that the tangential stresses $T_{22}$ and $T_{33}$ will be different on the two different sides of the wall, indicating that the static, planar domain wall solution is unstable against bending. The wall will tend to bend toward the higher energy density (i.e., higher $\left|T_{22}\right|=\left|T_{33}\right|$ ) side, and one expects a network of bubbles to form. A bubble is surrounded by a lower energy density region and encloses a higher energy density one. We are interested in the case where $\varphi_{1} \gg \varphi_{\min }$, that is, in the case where $\varphi_{\min }$ takes on a small value, giving rise to a small scale factor $B_{\min }=e^{\sqrt{\frac{2}{3} \kappa \varphi_{\min }}}$, and $\varphi_{1}$ takes on a very large value so that the scale factor $B_{1}=e^{\sqrt{\frac{2}{3}} \kappa \varphi_{1}}$ becomes large enough so that the size of the extra dimension, characterized by the circumference $L_{5}=(2 \pi R) B(x)$, becomes macroscopic. In this case, the "semi-vacuumless" domain bubble encloses a region that is effectively five dimensional and is surrounded by a region that is effectively four dimensional, or vice-versa. Here, attention is focused on the case where $U\left(\varphi_{1}\right)>U\left(\varphi_{\min }\right) \approx 0$ (which can be achieved by tuning the cosmological constant, for instance), so that, effectively, the bubble encloses a $5 \mathrm{~d}$ spacetime and is surrounded by a $4 \mathrm{~d}$ spacetime. Such a bubble, however, is not stable, since there is no mechanism to balance the inward pressure caused by the bubble's surface energy (and an assumed negligibly small volume energy), and the bubble therefore collapses.

\section{A STABILIZATION MECHANISM FOR A 4D DIMENSION BUBBLE ENCLOSING 5D}

\section{A. The Dimension Bubble as a Nontopological Soliton Entrapping Bosons}

The "dimension bubble" described above, which is effectively surrounded by a $4 \mathrm{~d}$ spacetime and encloses a $5 \mathrm{~d}$ spacetime, can be stabilized by a gas of particles trapped within the bubble. This mechanism can arise quite naturally if the Lagrangian $\mathcal{L}$ contains a complex scalar field $\chi$ with Lagrangian 


$$
\begin{aligned}
\mathcal{L}_{\chi} & =(2 \pi R) \mathcal{L}_{5, \chi}=\tilde{g}^{M N}\left(\partial_{M} \chi\right)^{*}\left(\partial_{N} \chi\right)-V \\
& =e^{\sqrt{\frac{2}{3}} \kappa \varphi} g^{\mu \nu}\left(\partial_{\mu} \chi\right)^{*}\left(\partial_{\nu} \chi\right)-V
\end{aligned}
$$

where $V=V(|\chi|)$ and we have used, from (四), $\tilde{g}^{\mu \nu}=e^{\sqrt{\frac{2}{3}} \kappa \varphi} g^{\mu \nu}$, along with the assumption $\partial_{5} \chi=0$. From (5), the associated $4 d$ Einstein Frame action is

$$
S_{\chi}=\int d^{4} x \sqrt{-g} e^{-\sqrt{\frac{2}{3}} \kappa \varphi} \mathcal{L}_{\chi}=\int d^{4} x \sqrt{-g} \mathcal{L}_{\chi, \text { eff }}
$$

from which we identify an effective $4 \mathrm{~d} \chi$ Lagrangian

$$
\mathcal{L}_{\chi, e f f}=|\partial \chi|^{2}-e^{-\sqrt{\frac{2}{3}} \kappa \varphi} V(|\chi|)
$$

and an effective $\chi$ potential

$$
U_{\chi}=e^{-\sqrt{\frac{2}{3}} \kappa \varphi} V(|\chi|)
$$

The $\chi$ boson mass obtained from the effective potential $U_{\chi}$ is

$$
m_{\chi}^{2}=e^{-\sqrt{\frac{2}{3}} \kappa \varphi}\left(\frac{\partial^{2} V}{\partial \chi^{*} \partial \chi}\right)_{v a c}=\mu^{2} e^{-\sqrt{\frac{2}{3}} \kappa \varphi}
$$

where $\mu^{2}=\left.\left(\partial^{2} V / \partial \chi^{*} \partial \chi\right)\right|_{\text {vacuum }}$ is the mass parameter in the $5 \mathrm{~d}$ theory.

For our dimension bubble enclosing a 5 d spacetime (with large $\varphi$ inside the bubble) and surrounded by a $4 \mathrm{~d}$ spacetime (with relatively small $\varphi$ outside the bubble), if the condition $\exp \left[\kappa\left(\varphi_{\text {out }}-\varphi_{\text {in }}\right)\right]=\exp \left[\kappa\left(\varphi_{1}-\varphi_{\text {min }}\right)\right] \ll 1$, then the $\chi$ boson masses inside and outside the bubble differ dramatically with

$$
\frac{m_{\chi, \text { out }}^{2}}{m_{\chi, \text { in }}^{2}}=e^{-\sqrt{\frac{2}{3}} \kappa\left(\varphi_{\text {out }}-\varphi_{\text {in }}\right)} \gg 1
$$

The result is that $\chi$ bosons get trapped inside the bubble, as with the case of nontopological soliton (NTS) bags studied previously [6]. The trapped $\chi$ bosons exert an outward pressure that, at equilibrium, can counterbalance the inward pressure of the bubble wall.

\section{B. $\chi$-Boson Stabilized Solitons}

Let us consider the case where the $\chi$ boson has a mass $m$ outside the bag and is effectively massless inside the bag. The ground state boson kinetic energy inside the bag can be estimated by setting the De Broglie wavelength of a single boson equal to the diameter of

the bag that confines it: $K E \approx p=\hbar k=2 \pi / \lambda \sim \pi / R$. For $Q$ bosons inside the bag, the kinetic energy is then estimated to be $E_{\chi} \sim Q \pi / R$, which agrees with the results obtained 
from use of a trial function by Frieman et al. (see ref [6]). We shall verify this result by obtaining the solution for $\chi$ in the $\varphi$ background and calculating explicitly the energy $E_{\chi}$.

For definiteness, we take the effective potential of (24) to be given by $U_{\chi}=m_{\chi}^{2} \chi^{*} \chi$, where $m_{\chi}=m$ outside the soliton and $m_{\chi}=0$ inside. For the background solution we assume $\varphi$ to take on constant values $\varphi_{i n}$ and $\varphi_{\text {out }}$ on the inside and outside of the soliton, with a jump at the bubble wall (i.e., the thin wall approximation). The effective Lagrangian $\mathcal{L}_{\chi}=|\partial \chi|^{2}-U_{\chi}$ then gives an associated energy density $T_{00}^{\chi}=\left|\partial_{0} \chi\right|^{2}+\left|\partial_{r} \chi\right|^{2}+U_{\chi}$ for a spherically symmetric soliton. The energy $E=E_{\chi}+E_{\text {wall }}$ of the soliton is then $E=4 \pi \int T_{00}^{\chi} r^{2} d r+4 \pi R^{2} \Sigma$, where $R$ is the soliton radius and $\Sigma$ is the surface energy density of the bubble wall. To evaluate $E$, we first need to obtain the solution for $\chi$.

We take $\chi(r, t)=F(r) e^{i \omega t}$. The field equation for $\chi$ gives

$$
\frac{d^{2} F}{d r^{2}}+\frac{2}{r} \frac{d F}{d r}+\left(\omega^{2}-m_{\chi}^{2}\right) F=0
$$

and the conserved charge of $Q$ bosons trapped inside the soliton is

$$
Q=8 \pi \omega \int_{0}^{\infty} F^{2} r^{2} d r
$$

For a finite energy solution, we require $F$ to vanish outside the bubble for the case that $\left(\omega^{2}-m^{2}\right) \leq 0$, while for $\left(\omega^{2}-m^{2}\right)>0$ we have

$$
F \propto \frac{e^{-\left(m^{2}-\omega^{2}\right)^{1 / 2} r}}{\left(m^{2}-\omega^{2}\right)^{1 / 2} r}
$$

so that $F$ rapidly approaches zero outside the soliton. We therefore take $F=0$ as an approximate solution for $r>R$. The regular solution for $F$ inside the soliton is $F=$ $F_{0} \sin (\omega r) / \omega r$. The approximate solution is therefore

$$
F=\left\{\begin{array}{cc}
F_{0} \frac{\sin (\omega r)}{\omega r}, & (r<R) \\
0, & (r>R)
\end{array}\right\}
$$

For continuity of the solution at the bubble wall $r=R$, we set

$$
\omega R=\pi
$$

The solution (30) and the boundary condition (31) give the energy $E_{\chi}=4 \pi^{2} F_{0}^{2} / \omega$ and boson charge $Q=4 \pi^{2} F_{0}^{2} / \omega^{2}$, or

$$
E_{\chi}=\omega R=\frac{Q \pi}{R}
$$

as obtained in the quick estimate above. 
We have assumed the vacuum energy density to vanish inside the soliton, so that (neglecting gravitational effects) the energy of the NTS is determined by the contributions from the domain wall surface energy and the energy of the entrapped bosons,

$$
E=\frac{Q \pi}{R}+4 \pi \Sigma R^{2}
$$

where $\Sigma$ is the surface energy density of the wall. Minimization of this energy gives the NTS equilibrium radius

$$
R=\frac{1}{2}\left(\frac{Q}{\Sigma}\right)^{1 / 3}
$$

From (33) and (34) the equilibrium energy, or mass, of the bubble is

$$
E=3 \pi\left(Q^{2} \Sigma\right)^{1 / 3}
$$

We can obtain approximate stability conditions for the bosonic charge $Q$ and the bubble radius $R$ by requiring the $\chi$ bosons to remain trapped inside the bubble rather than escaping to the outside. For $Q$ free bosons of mass $m$ outside the bag, the minimal energy is $E_{Q \text { free }}=$ $Q m$. Therefore, for the NTS bubble stability we require the equilibrium bubble energy $E<E_{Q \text { free }}$ which, by (35), implies that

$$
Q^{1 / 3}>3 \pi \frac{\Sigma^{1 / 3}}{m}
$$

By (34) this translates into the condition for the bubble radius that

$$
R>\frac{3 \pi}{2 m}
$$

for a stable bubble.

\section{SUMMARY}

We have considered a type of case similar to that previously studied by Blau and Guendelman [1] wherein a scalar field (appearing within a dimensionally reduced $4 \mathrm{~d}$ effective theory)-that is associated with the scale factor of an extra (compact) dimension in a $5 \mathrm{~d}$ theory-forms a domain wall interpolating between two different spatial regions. When the scalar field takes on radically different values in the two different domains, the effective dimensionality of the space also becomes different in these different domains. [As an example, we have followed [1] in constructing a form of $4 \mathrm{~d}$ effective potential arising from the low temperature Rubin-Roth potential [2] (due to one loop quantum contributions from massless bosons and massive or massless fermions) along with a cosmological constant.] Domain 
walls which are unstable against bending can give rise to a network of closed "dimension bubbles", where the effective spacetime dimensionality is different in the interior and exterior regions of a bubble. Without a stabilization mechanism, these bubbles collapse due to the tension in the bubble walls.

However, the inclusion of a scalar field $\chi$ in the original 5 d theory can give rise to a stabilization mechanism in a rather natural way. In essence, due to the different values of the conformal factor in the interior and exterior regions of the bubble, the $\chi$ boson mass can change drastically across the bubble wall. We have considered the case of a bubble surrounded by a $4 \mathrm{~d}$ region and enclosing a $5 \mathrm{~d}$ region, with $\chi$ bosons that are massive outside the bubble, but are effectively massless inside the bubble. Therefore, the $\chi$ particles are effectively trapped inside the bubble, exerting an outward pressure which can stabilize the bubble from total collapse, provided that certain model parameters lie within appropriate ranges. This resembles the stabilization mechanism for "ordinary" 4 d nontopological solitons (NTSs) studied previously by Frieman, Gleiser, Gelmini and Kolb [6].

Semi-vacuumless domain walls and dimension bubbles can also arise from the $4 \mathrm{~d}$ effective potential associated with the model of a classical stabilization of two extra dimensions in a spherically reduced 6d model described by Carroll, Geddes, Hoffman and Wald [7] (see sec. 3 of that paper). In that model, an extra dimensional magnetic field, along with a cosmological constant and the curvature term of the extra dimensional two-sphere, give rise to a $4 \mathrm{~d}$ effective potential [given by eq.(36) of ref. [7]] with the same basic shape as the effective potential $U$ arising from quantum fluctuations considered here [see eq. (9) above]. These bubbles could also be stabilized by the same mechanism of $\chi$ boson entrapment.

As in the case of the ordinary NTSs, dimension bubbles, if they exist, could contribute to the dark matter of our (mostly) 4d universe. 
[1] S.K. Blau and E.I. Guendelman, Phys. Rev. D40, 1909 (1989)

[2] M.A. Rubin and B.D. Roth, Phys. Lett. 127B, 55 (1983)

[3] T. Appelquist and A. Chodos, Phys. Rev. Lett. 50, 141 (1983)

[4] I.Cho and A. Vilenkin, Phys. Rev. D59, 021701 (1999)

[5] D. Bazeia, Phys. Rev. D60, 067705 (1999)

[6] J.A. Frieman, G.B. Gelmini, M. Gleiser and E.W. Kolb, Phys. Rev. Lett. 23, 2101 (1988)

[7] S.M. Carroll, J. Geddes, M.B. Hoffman and R.M. Wald, Phys. Rev. D66, 024036 (2002) 